\title{
Blood Cell Count Derived Inflammation Indexes in Patients with Idiopathic Pulmonary Fibrosis
}

\author{
Angelo Zinellu ${ }^{1} \cdot$ Panagiotis Paliogiannis $^{2}$ (D) Elisabetta Sotgiu ${ }^{1} \cdot$ Sabrina Mellino ${ }^{1} \cdot$ Arduino A. Mangoni ${ }^{4}$. \\ Elisabetta Zinellu ${ }^{3} \cdot$ Silvia Negri ${ }^{2,3}$. Claudia Collu ${ }^{2,3} \cdot$ Gianfranco Pintus $^{1,5} \cdot$ Antonello Serra $^{6}$. \\ Angelo Maria Pistuddi ${ }^{6}$. Ciriaco Carru ${ }^{1} \cdot$ Pietro Pirina ${ }^{2,3} \cdot$ Alessandro G. Fois ${ }^{2,3}$
}

Received: 24 May 2020 / Accepted: 10 August 2020 / Published online: 25 August 2020

(c) The Author(s) 2020

\begin{abstract}
Purpose Inflammation and immunity play a pivotal but yet unclear role in idiopathic pulmonary fibrosis (IPF), a chronic disorder characterized by progressive damage of lung parenchyma and severe loss of lung function despite optimal treatment. However, the pathophysiological and predictive role of combined blood cell count indexes of inflammation in IPF is uncertain. Methods Seventy-three patients with IPF and 62 healthy subjects matched for age, gender and smoking status were included in this cross-sectional study.

Results We found significant differences in neutrophil to lymphocyte ratio (NLR), derived neutrophil to lymphocyte ratio (dNLR), monocyte to lymphocyte ratio (MLR), platelet to lymphocyte ratio (PLR), systemic inflammation response index (SIRI) and aggregate index of systemic inflammation (AISI) between IPF patients and healthy controls. In logistic regression, all combined blood inflammation indexes, barring PLR, were independently associated with the presence of IPF after adjusting for age, gender, body mass index and smoking status. Furthermore, significant associations between FVC\% and NLR, LMR, SIRI and AISI, and between DLCO \% and NLR, dNLR, LMR, SIRI and AISI, were observed.

Conclusions In conclusion, our data indicate significant alterations of combined blood cell count indexes of inflammation in IPF.
\end{abstract}

Keywords Idiopathic pulmonary fibrosis $\cdot$ IPF $\cdot$ Inflammation $\cdot$ LMR $\cdot$ NLR $\cdot$ PLR

Panagiotis Paliogiannis

panospaliogiannis@gmail.com

1 Department of Biomedical Sciences, University of Sassari, Sassari, Italy

2 Department of Medical, Surgical and Experimental Sciences, University of Sassari, Sassari, Italy

3 Unit of Respiratory Diseases, University Hospital Sassari (AOU), Sassari, Italy

4 Department of Clinical Pharmacology, College of Medicine and Public Health, Flinders University and Flinders Medical Centre, Adelaide, Australia

5 Department of Medical Laboratory Sciences, College of Health Sciences and Sharjah Institute for Medical Research, University of Sharjah, P.O. Box: 27272, Sharjah, United Arab Emirates

6 Unit of Occupational Medicine, University Hospital Sassari (AOU), Sassari, Italy

\section{Introduction}

Idiopathic pulmonary fibrosis (IPF) is a chronic progressive fibrosing interstitial lung disease of unknown aetiology, occurring mainly in adults, that is characterized by a histopathologic and/or radiologic pattern of usual interstitial pneumonia [1]. The progressive accumulation of fibrotic tissue leads to irreversible lung damage. As a consequence, the estimated survival of untreated patients is limited, 2-3 years [2]. It is commonly accepted that fibrogenesis in IPF results from an aberrant repair process due to recurrent microinjury of alveolar epithelial cells [3-5]. The exaggerated wound repair and tissue remodelling lead to chronic inflammation and, ultimately, fibrosis. Both innate and adaptative responses seem to be involved in this process [6]. The excessive inflammatory response in IPF is multifactorial although the transforming growth factor- $\beta$ (TGF- $\beta$ ) signalling is thought to play a key role [7]. It is well known that inflammation involves a highly coordinated network 
of immune-related cells, such as neutrophils, lymphocytes and monocytes [8]. Platelets can also modulate inflammation through direct cell-cell communications and the production of inflammatory mediators [9].

There is good evidence that indexes derived from calculating the ratios between neutrophils, lymphocytes, monocytes and platelets counts are more strongly associated with chronic inflammation conditions when compared with individual cell populations [8]. Indeed, combined blood cell count indexes of systemic inflammation have gained relevant scientific interest over the last decade. The neutrophil to lymphocyte ratio (NLR), derived neutrophil to lymphocyte ratio (dNLR), monocyte to lymphocyte ratio (MLR), platelet to lymphocyte ratio (PLR), systemic inflammation response index (SIRI) and aggregate inflammation systemic index (AISI) are increasingly being investigated as markers of inflammation in several disorders [10-14], including lung disease states such as COPD [15, 16] and asthma [17]. However, no information is currently available regarding possible associations between combined blood cell count indexes of inflammation and IPF. We sought to address this issue by measuring NLR, dNLR, PLR, LMR SIRI and AISI in IPF patients and in controls and investigating associations with clinically relevant parameters of lung function, $\mathrm{FEV}_{1}$ (Forced Expiratory Volume in the 1st second), FVC (Forced Vital Capacity) and DLCO (Diffusion Capacity for Carbon Monoxide).

\section{Materials and Methods}

This was a case-control study of 73 consecutive newly diagnosed IPF patients recruited at the Respiratory Unit of the University of Sassari between 2016 and 2019. All patients signed a written consent and the study was approved by the ethics committee of the University Hospital of Cagliari (2262/CE-17/11/2015). IPF was diagnosed according to the current evidence-based guidelines for the diagnosis and management of IPF [18]. High-resolution computed tomography (HRCT) images and lung-biopsy samples of individual patients were reviewed by two experienced radiologists and two experienced pathologists, respectively. Each diagnosis was confirmed during multidisciplinary meetings attended by local respiratory, pathology, and radiology experts in interstitial lung disease. Measurements of $\mathrm{FEV}_{1}$, FVC and DLCO, expressed as percentages of predicted values $\left(\% \mathrm{FEV}_{1}, \% \mathrm{FVC}\right.$ and $\% \mathrm{DLCO}$, respectively), were performed in accordance with the criteria published by the American Thoracic Society and the European Respiratory Society [19]. Patients with the following conditions were excluded from the study: patients currently experiencing an acute exacerbation of IPF; comorbid conditions including malignancy, bleeding tendency, and severe hepatic or renal dysfunction; use of immunosuppressants, antifibrotic drugs including interferon, D-penicillamine, and colchicine, or oral corticosteroids during the preceding 3 months. A group of 63 healthy controls matched for age, sex and smoking status, with no medical history, was also included.

Fasting blood samples from IPF patients and matched controls were respectively collected at first hospital admission in the Unit of Respiratory Diseases, and the Unit of Occupational Medicine of the University Hospital of Sassari (AOU Sassari); blood cell counts were performed using an automatic blood counter CellDyn Sapphire (Abbott Diagnostics, Santa Clara, CA, USA) in a certified laboratory. The following combined indexes were evaluated: NLR, dNLR, LMR, PLR, SIRI and AISI. The SIRI was calculated by multiplying the number of neutrophils with that of monocytes and dividing the product by the number of lymphocytes [20]. The AISI was calculated by multiplying the number of neutrophils, monocytes and platelets and dividing the product by the number of lymphocytes [10].

Results are expressed as mean values (mean $\pm \mathrm{SD}$ ) or median values (median and interquartile range, IQR). Variables distribution was assessed by the Shapiro-Wilk test. Statistical differences between groups were evaluated using unpaired Student's t-test or Mann-Whitney rank sum test, as appropriate. Differences between categorical variables were assessed by Chi-squared test. Correlations between variables were assessed by Pearson's correlation or Spearman's correlation as appropriate. Logistic regression analysis was performed to evaluate independent associations between IPF disease and combined blood cell count indexes of inflammation. The association between different indexes and IPF was evaluated using receiver operating characteristics (ROC) curve analysis and selection of optimal cut-off values for sensitivity and specificity according to the Youden Index. Statistical analyses were performed using MedCalc for Windows, version 15.464 bit (MedCalc Software, Ostend, Belgium).

\section{Results}

Table 1 describes the clinical characteristics, lung functional parameters, and blood cell counts of IPF patients and healthy controls. As expected, $\mathrm{FEV}_{1}(\%)$ and $\mathrm{FVC}(\%)$ were significantly lower in IPF patients than in controls (mean $81 \pm 22 \%$ vs $92 \pm 18 \%, p<0.001$; median $72 \%$; IQR $58-90 \%$ vs $92 \%$; IQR $84-105 \% p<0.001$, respectively). IPF patients had higher WBCs (median $8.7 \times 10^{9} \mathrm{~L}$; IQR $7.3-10.3 \times 10^{9} \mathrm{~L}$ vs $7.2 \times 10^{9} \mathrm{~L}$; IQR $\left.6.4-8.7 \times 10^{9} \mathrm{~L}, p<0.001\right)$ and neutrophils (median $5.5 \times 10^{9} \mathrm{~L}$; IQR $4.6-6.7 \times 10^{9} \mathrm{~L}$ vs $4.1 \times 10^{9} \mathrm{~L}$; IQR 3.5-5.4 $\left.\times 10^{9} \mathrm{~L}, p<0.001\right)$ and lower lymphocyte values (median $2.0 \times 10^{9} \mathrm{~L}$; IQ $1.5-2.4 \times 10^{9} \mathrm{~L}$ vs $2.3 \times 10^{9} \mathrm{~L}$; IQR $\left.1.9-2.6 \times 10^{9} \mathrm{~L}, p<0.05\right)$. There were no significant 
Table 1 Demographic and functional parameters and blood count results of healthy subjects and IPF patients

\begin{tabular}{lll}
\hline & $\begin{array}{l}\text { Controls } \\
(n=62)\end{array}$ & $\begin{array}{l}\text { IPF } \\
(n=73)\end{array}$ \\
\hline Age $($ years $)$ & $67(66-69)$ & $70(64-75)$ \\
Gender $(\mathrm{F} / \mathrm{M})$ & $19 / 43$ & $22 / 51$ \\
BMI $\left(\mathrm{kg} / \mathrm{m}^{2}\right)$ & $27.1 \pm 4.0$ & $27.2 \pm 3.9$ \\
Smokers, never/current/ex $(\%)$ & $30 / 10 / 60$ & $19 / 6 / 75$ \\
FEV $(\%$ predicted $)$ & $99 \pm 18$ & $81 \pm 22^{* * *}$ \\
FVC $(\%$ predicted $)$ & $92(84-105)$ & $72(58-90)^{* * *}$ \\
TLC $(\%$ predicted $)$ & - & $75 \pm 17$ \\
DLCO $(\%$ predicted $)$ & - & $55 \pm 23$ \\
6MWT $(\mathrm{m})$ & - & $342 \pm 206$ \\
GAP index $(1 / 2 / 3 / 4 / 5 / 6 / 7 \%)$ & - & $4 / 19 / 25 / 24 / 17 / 8 / 3$ \\
Disease stage $(\mathrm{I} / \mathrm{II} / \mathrm{III} \%)$ & - & $48 / 42 / 10$ \\
WBC $\left(\times 10^{9} \mathrm{~L}\right)$ & $7.2(6.4-8.7)$ & $8.7(7.3-10.3)^{* * *}$ \\
Monocytes $\left(\times 10^{9} \mathrm{~L}\right)$ & $0.5(0.39-0.60)$ & $0.5(0.40-0.70)$ \\
Lymphocytes $\left(\times 10^{9} \mathrm{~L}\right)$ & $2.3(1.9-2.6)$ & $2.0(1.5-2.4)^{*}$ \\
Neutrophils, $\left(\times 10^{9} \mathrm{~L}\right)$ & $4.1(3.5-5.4)$ & $5.5(4.6-6.7)^{* * *}$ \\
Platelet $\left(\times 10^{9} \mathrm{~L}\right)$ & $242 \pm 60$ & $242 \pm 68$ \\
\hline
\end{tabular}

Data are presented as mean \pm standard deviation or median (interquartile range)

FEV1 forced expiratory volume in the 1st second, $F V C$ forced vital capacity, $T L C$ total lung capacity, $D L C O$ diffusion capacity for carbon monoxide, GAP gender, age and two lung physiology variables index, $P 6 M W T$ six-minute walk test, $W B C$ white blood cell

${ }^{*} p<0.05, * * p<0.01, * * * p<0.001$

between-group differences in BMI, monocyte and platelet values.

The combined indexes data are described in Table 2. NLR (median 2.67; IQR 1.89-2.67 vs 1.87; IQR 1.52-2.35, $p<0.001$ ), d-NLR (median 1.88; IQR 1.33-2.26 vs 1.31; IQR $1.15-1.70, p<0.001$ ), PLR (mean $132 \pm 74$ vs $110 \pm 36$,

Table 2 Complete blood cell count-derived indexes in idiopathic pulmonary fibrosis and healthy subjects

\begin{tabular}{lll}
\hline & Controls (62) & IPF (73) \\
\hline NLR & $1.87(1.52-2.35)$ & $2.67(1.89-2.67)^{* * * *}$ \\
dNLR & $1.31(1.15-1.70)$ & $1.88(1.33-2.26)^{* * *}$ \\
LMR & $5.10 \pm 1.71$ & $4.05 \pm 1.75 \mathrm{c}$ \\
PLR & $110 \pm 36$ & $132 \pm 74^{*}$ \\
SIRI & $0.89(0.57-1.26)$ & $1.49(0.89-2.40)^{* * *}$ \\
AISI & $198(128-316)$ & $335(183-550)^{* * *}$ \\
\hline
\end{tabular}

Data are presented as mean \pm standard deviation or median (interquartile range)

$N L R$ neutrophil to lymphocyte, $d N L R$ derived NLR, LMR lymphocyte to monocyte, PLR platelet to lymphocyte, SIRI Systemic Inflammation Response Index, AISI aggregate index of systemic inflammation, $I P F$ idiopathic pulmonary fibrosis

$* p<0.05, * * p<0.01, * * * p<0.001$ $p<0.05$ ), SIRI (median 1.49; IQR 0.89-2.40 vs 0.89; IQR $0.57-1.26, p<0.001$ ) and AISI (median 335; IQR 183-550 vs 198; IQR 128-316, $p<0.001$ ) were significantly higher in IPF patients whereas the LMR was significantly lower (mean: $4.05 \pm 1.75$ vs $5.10 \pm 1.71, p<0.001$ ). In univariate logistic regression, crude ORs for all inflammation combined indexes were significant, as reported in Table 3 . In multivariate logistic regression, the ORs for NLR $(\mathrm{OR}=2.633$; 95\% CI 1.604-4.322, $p=0.0001)$, d-NLR (OR $=3.941$; 95\% CI 1.851-8.390, $p=0.0004)$, LMR (OR $=0.685$; $95 \%$ CI $0.543-0.865, p=0.0015)$, SIRI $(\mathrm{OR}=3.840 ; 95 \% \mathrm{CI}$ $1.906-7.736, p=0.0002)$ and AISI $(\mathrm{OR}=1.003 ; 95 \% \mathrm{CI}$ $1.001-1.005, p=0.0045)$ remained significant after adjusting for age, gender, BMI and smoking status.

In IPF patients, combined blood cell count indexes of inflammation were also significantly associated with functional lung parameters (Table 4). In particular, $\mathrm{FEV}_{1}$ was negatively associated with NLR (rho $=-0.24, p=0.04)$, SIRI (rho $=-0.27, p=0.019$ ) and AISI (rho $=-0.26$, $p=0.03)$, FVC $\%$ was negatively associated with NLR (rho $=-0.32, p=0.007)$, SIRI $($ rho $=-0.30, p=0.012)$ and AISI (rho $=-0.24 p=0.04)$ and positively related with LMR (rho $=0.27, p=0.02)$, and DLCO \% was negatively associated with NLR (rho $=-0.40, p=0.003)$, dNLR (rho $=-0.33, p=0.015)$, SIRI (rho $=-0.35, p=0.01)$ and AISI (rho $=-0.16, p=0.26$ ), and positively associated with LMR (rho $=0.28, p=0.04$ ). By contrast, barring LMR and SIRI, there were no significant associations between combined blood cell count indexes and functional lung parameters in healthy controls.

Table 3 Univariate and multivariate logistic regression for blood cell count-derived indexes

\begin{tabular}{llll}
\hline & & $95 \%$ CI & \\
\hline Crude OR & & & \\
NLR & 2.851 & $1.748-4.649$ & $<0.0001$ \\
dNLR & 4.486 & $2.126-9.466$ & 0.0001 \\
LMR & 0.697 & $0.559-869$ & 0.0013 \\
PLR & 1.008 & $1.000-1.015$ & 0.043 \\
SIRI & 3.793 & $1.972-7.297$ & 0.0001 \\
AISI & 1.003 & $1.001-1.005$ & 0.0014 \\
Corrected OR & & & \\
NLR & 2.633 & $1.604-4.322$ & 0.0001 \\
dNLR & 3.941 & $1.851-8.390$ & 0.0004 \\
LMR & 0.685 & $0.543-0.865$ & 0.0015 \\
PLR & 1.007 & $0.999-1.015$ & 0.10 \\
SIRI & 3.840 & $1.906-7.736$ & 0.0002 \\
AISI & 1.003 & $1.001-1.005$ & 0.0045 \\
\hline
\end{tabular}

$N L R$ neutrophil to lymphocyte, $d N L R$ derived NLR, $L M R$ lymphocyte to monocyte, PLR platelet to lymphocyte, SIRI Systemic Inflammation Response Index, AISI aggregate index of systemic inflammation, $O R$ odds ratio, $C I$ confidence interval 
Table 4 Correlations between blood cell count indexes of systemic inflammation and functional parameters in patients with IPF and controls

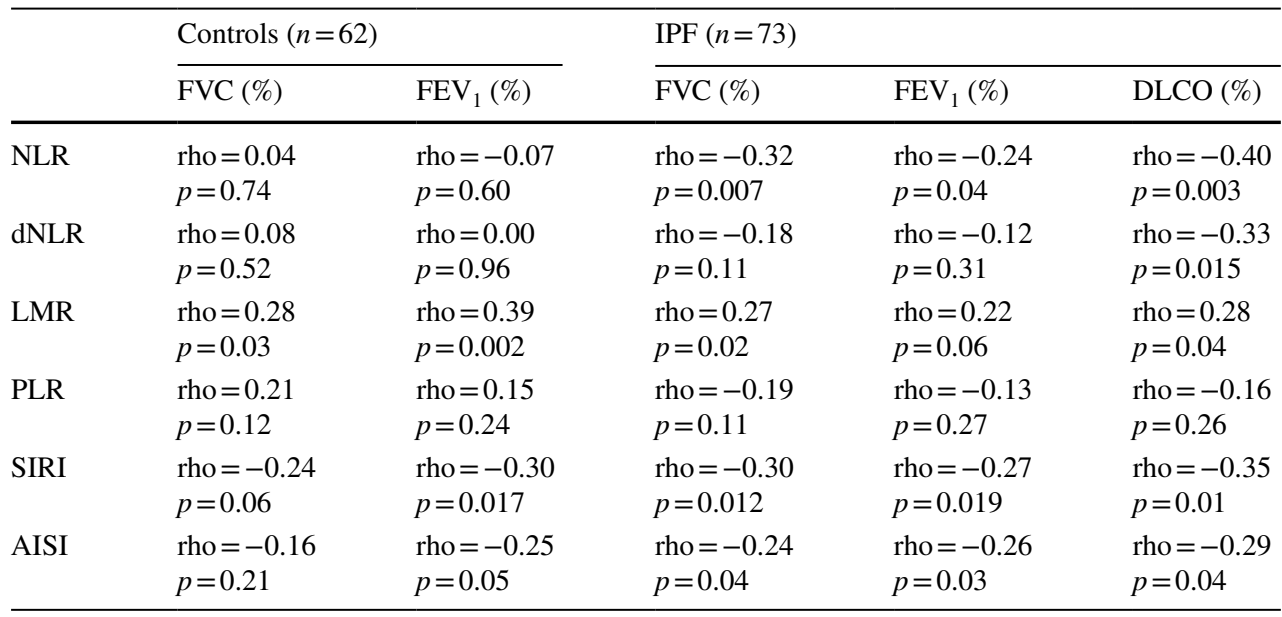

$N L R$ neutrophil to lymphocyte, $d N L R$ derived NLR, $L M R$ lymphocyte to monocyte, $P L R$ platelet to lymphocyte, SIRI Systemic Inflammation Response Index, AISI Aggregate index of systemic inflammation, $I P F$ idiopathic pulmonary fibrosis, $F V C$ forced vital capacity, FEVI forced expiratory volume in the 1 st second, $D L C O$ diffusion capacity for carbon monoxide
ROC curve analysis was carried out to evaluate the sensitivity, specificity, and the diagnostic accuracy of the combined inflammation indexes in distinguishing IPF patients from healthy subjects (Table 5). Except for PLR, all AUC values were significant. The better performing index was the NLR, with a threshold of $2.545,55 \%$ sensitivity, and $85 \%$ specificity (AUC $=0.735,95 \%$ CI $0.652-0.807 p<0.001)$.

\section{Discussion}

We observed significant differences in neutrophil to lymphocyte ratio (NLR), derived neutrophil to lymphocyte ratio (dNLR), monocyte to lymphocyte ratio (MLR), platelet to lymphocyte ratio (PLR), systemic inflammation response index (SIRI) and aggregate index of systemic inflammation (AISI) between patients with IPF and a group of healthy controls matched for age, gender and smoking status. All combined blood inflammation indexes, except for PLR, were independently associated with IPF after adjusting for age, gender, body mass index and smoking status. Furthermore, there were significant associations between $\mathrm{FVC} \%$ and NLR, LMR, SIRI and AISI, and between DLCO $\%$ and NLR, dNLR, LMR, SIRI and AISI.

IPF is the most severe and frequent form of interstitial lung disease, characterized by aberrant deposition of extracellular matrix due to recurrent micro-injuries to the alveolar epithelium. Mediators released by epithelial cells activate fibroblast proliferation leading to accumulation of fibroblasts and highly active myofibroblasts. These cells are resistant to apoptosis and lead to extensive lung remodelling, through deposition of extracellular matrix components such as hyaluronan, fibronectin and interstitial collagens, and consequent alveolar wall thickening and impaired gas exchange $[6,21]$. While the exact aetiology of IPF remains unknown, several lines of evidence indicate that all stages of fibrosis are accompanied by innate and adaptive response, although treatment that modulates inflammation, e.g. steroids, has been shown to be ineffective, or even harmful, in clinical trials [22-24]. Although this has led to the proposition that inflammation may represent an epiphenomenon, rather than
Table 5 Receiver operating characteristics (ROC) curves and prognostic accuracy of blood cell count-derived indexes

\begin{tabular}{llllllc}
\hline & AUC & $95 \%$ CI & $p$-value & Cut-off & $\begin{array}{l}\text { Sensibility } \\
(\%)\end{array}$ & Specificity (\%) \\
\hline NLR & 0.735 & $0.652-0.807$ & $<0.0001$ & $>2.545$ & 55 & 85 \\
dNLR & 0.714 & $0.630-0.788$ & $<0.0001$ & $>1.862$ & 53 & 85 \\
LMR & 0.663 & $0.576-0.742$ & 0.0004 & $<2.857$ & 29 & 100 \\
PLR & 0.590 & $0.502-0.674$ & 0.07 & $>113$ & 56 & 66 \\
SIRI & 0.729 & $0.646-0.802$ & $<0.0001$ & $>1.333$ & 58 & 85 \\
AISI & 0.685 & $0.599-0.762$ & 0.0001 & $>272$ & 60 & 71 \\
\hline
\end{tabular}

$N L R$ neutrophil to lymphocyte, $d N L R$ derived NLR, $L M R$ lymphocyte to monocyte, PLR platelet to lymphocyte, SIRI Systemic Inflammation Response Index, AISI Aggregate index of systemic inflammation, $A U C$ area under the curve, $C I$ confidence interval 
a key driver in IPF, the active involvement of immune cells in the pathophysiology of the disease is well accepted.

Recent findings suggest that neutrophil elastase (NE) promotes TGF- $\beta$ activation, fibroblast proliferation and myofibroblast differentiation, all contributing to enhance fibrosis [25]. The same study, according to previous observations [26], demonstrated that $\mathrm{NE}$ antagonists attenuate fibrosis both in asbestos and bleomycin induced fibrosis models, thus confirming the pro-fibrotic role of NE in IPF. Monocytes also play a crucial role during fibrogenesis as progenitor cells for pro-fibrotic macrophages and fibrocytes and by releasing pro-fibrotic inflammatory cytokines [7]. Furthermore, macrophages exhibit tissue-regenerating and pro-fibrotic function, depending on the local cytokine environment. In particular, M2 macrophages seem to have a central role in fibrosis regulation [27] acute exacerbations of IPF [28]. In addition, adaptive immunity cells Th1 and Th17 promote pulmonary fibrosis, whereas $\mathrm{Th} 2$ and $\mathrm{Th} 22$ cells inhibit fibrosis. Similarly, regulatory T cells (Tregs) and Th 9 cells exhibit both pro- and anti-fibrotic characteristics [29]. These observations strongly justify the investigation of blood cell count derived inflammation indexes in IPF.

Our results show significant alterations of these indexes in IPF patients, thus supporting the ability of these markers to detect systemic inflammation also in this group. These data agree with previous studies demonstrating that NLR and PLR are reliable indexes of systemic inflammation and predict the prognosis of several chronic inflammatory diseases [10, 11, 15-17]. In logistic regression, except for PLR, all combined blood inflammation indexes were independently associated with the presence of IPF after adjusting for age, gender, BMI and smoking status. Moreover, they were significantly associated with robust functional lung parameters. In particular, the association between $\mathrm{FVC} \%$ and NLR, LMR, SIRI and AISI and between DLCO $\%$ and NLR, dNLR, LMR, SIRI and AISI indicate a strong relationship between these inflammation indexes and disease severity. These data agree with previous observations in which the concentration of inflammatory biomarkers, such as BALF IL-8, was significantly associated with a reduced FVC\% and DLCO\% [30], and the extent of sputum neutrophilia was correlated with FVC\% in IPF patients [31]. Furthermore, Tsoutsou et al. [32] found that serum concentrations of ICAM-2 (a circulating adhesion molecule with immunomodulatory effects) were negatively associated with DLCO\%. Daniil et al. [33] also that reported that tissue CD8 + TLs inversely correlated with FVC\%, TLC\% and DLCO $\%$ and that CD68 + cells negative correlated with both $\mathrm{FVC} \%$ and $\mathrm{FEV} 1 \%$.

In ROC curve analysis, the NLR, dNLR, LMR, SIRI and AISI were able to significantly discriminate patients with IPF from healthy subjects. The diagnostic accuracy was higher (AUC > 0.7) with NLR, dNLR and SIRI. NLR was the index which performed better, even than the combined indexes (SIRI and AISI) which included more than two blood cell populations in their calculation. The rationale for the use of such indexes, is based on the fact that multiple blood cell populations participate in systemic inflammation as we mentioned before, and therefore, inclusion of these populations in the calculation of an index should better reflect the inflammatory state and improve its predictive abilities. Indeed, the activation of neutrophils during systemic inflammation, not only increases their number, but stimulates also the production of several mediators (like IL-1a), which in turn stimulate megakaryocytes to produce platelets [34]; in addition, specific negligible fractions of monocytes in steady-state conditions have been reported to consistently increase during inflammation $[35,36]$. Therefore, AISI or SIRI are hypothesized to perform better than more restricted indexes. Nevertheless, our study showed that both platelet and monocytes are not different between IPF patients and healthy controls, and combined indexes do not perform better than NLR. The latters have shown better predictive abilities than NLR, in acute clinical conditions and malignancies $[10,14,37,38]$; this probably means that in cases of chronic inflammation adaptive phenomena prevent the increment of specific other than neutrophil and lymphocyte cell populations and stabilize their turnover. As a result, combined indexes may not be more effective than NLR in chronic systemic inflammation. The issue needs further evaluation. In addition, given the relatively low specificity of all the indexes tested in this study towards individual disease states, additional studies are required to determine their role in diagnosis, risk stratification, and prognosis, and their capacity to discriminate between IPF and other forms of interstitial lung diseases [15-17].

Our study has some limitations. In particular, the relatively small sample size and the single centre nature of the study, even accounting for the low prevalence of the disease, limits its generalizability. Furthermore, the cross-sectional design did not allow establishing a definite cause-effect relationship between the studied indexes, the presence of IPF, and the lung function parameters and no correlations were analysed between these indexes and relevant clinical parameters, such as dyspnoea and the six-minute walking test. These issues notwithstanding, our data show the presence of significant alterations in NLR, dNLR, LMR, SIRI and AISI values in IPF patients when compared to healthy controls, and clinically relevant associations with lung function parameters. Such indexes might be attractive from a clinical point of view as their calculation is relatively simple, inexpensive, and part of routinely available laboratory investigations. Nevertheless, large, prospective studies are now required to investigate their prognostic role in IPF. 
Funding This research was supported by grants from the Sardinian Fondo di Sviluppo e Coesione (FSC) 2014-2020, Patto per lo Sviluppo della Regione Sardegna, L.R.7-2017 (Grant No. RASSR82005) and Fondo di Ateneo per la Ricerca- annualità 2019. Open access funding provided by Università degli Studi di Sassari within the CRUI-CARE Agreement.

Data Availability Data can be provided by the corresponding author upon reasonable request.

Code availability Not applicable.

\section{Compliance with Ethical Standards}

Conflict of interest The authors declare that they have no conflict of interest.

Ethical Approval The study was approved by the ethics committee of the University Hospital of the University of Cagliari (2262/ CE-17/11/2015).

Consent to Participate Informed consent was obtained from all individual participants included in the study.

Open Access This article is licensed under a Creative Commons Attribution 4.0 International License, which permits use, sharing, adaptation, distribution and reproduction in any medium or format, as long as you give appropriate credit to the original author(s) and the source, provide a link to the Creative Commons licence, and indicate if changes were made. The images or other third party material in this article are included in the article's Creative Commons licence, unless indicated otherwise in a credit line to the material. If material is not included in the article's Creative Commons licence and your intended use is not permitted by statutory regulation or exceeds the permitted use, you will need to obtain permission directly from the copyright holder. To view a copy of this licence, visit http://creativecommons.org/licenses/by/4.0/.

\section{References}

1. Raghu G, Rochwerg B, Zhang Y et al (2015) An official ATS/ ERS/JRS/ALAT clinical practice Guideline: treatment of idiopathic pulmonary fibrosis. An update of the 2011 clinical practice guideline. Am J Respir Crit Care Med 192:e3-19

2. du Bois RM (2012) An earlier and more confident diagnosis of idiopathic pulmonary fibrosis. Eur Respir Rev 21:141-146

3. Selman M, Pardo A (2002) Idiopathic pulmonary fibrosis: an epithelial/fibroblastic cross-talk disorder. Respir Res 3:3

4. Selman M, King TE, Pardo A et al (2001) (2001) Idiopathic pulmonary fibrosis: prevailing and evolving hypotheses about its pathogenesis and implications for therapy. Ann Intern Med 134:136-151

5. Wuyts WA, Agostini C, Antoniou KM et al (2013) The pathogenesis of pulmonary fibrosis: a moving target. Eur Respir J 41:1207-1218

6. Wynn TA (2011) Integrating mechanisms of pulmonary fibrosis. J Exp Med 208:1339-1350

7. Heukels P, Moor CC, von der Thüsen JH et al (2019) Inflammation and immunity in IPF pathogenesis and treatment. Respir Med 147:79-91

8. Nathan C (2006) Neutrophils and immunity: challenges and opportunities. Nat Rev Immunol 6:173-182
9. Karhausen J, Choi HW, Maddipati KR et al (2020) Platelets trigger perivascular mast cell degranulation to cause inflammatory responses and tissue injury. Sci Adv 6(12):eaay6314

10. Paliogiannis P, Scognamillo F, Bellomo M et al (2015) Neutrophil to lymphocyte ratio as a predictor of thyroid papillary carcinoma. Acta Med Mediterr 31:371-375

11. Paliogiannis P, Satta R, Deligia G et al (2019) Associations between the neutrophil-to-lymphocyte and the platelet-to-lymphocyte ratios and the presence and severity of psoriasis: a systematic review and meta-analysis. Clin Exp Med 19:37-45

12. Erre GL, Paliogiannis P, Castagna F et al (2019) Meta-analysis of neutrophil-to-lymphocyte and platelet-to-lymphocyte ratio in rheumatoid arthritis. Eur J Clin Invest 49:e13037

13. Pinna A, Porcu T, D'Amico-Ricci G et al (2019) Complete blood cell count-derived inflammation biomarkers in men with agerelated macular degeneration. Ocul Immunol Inflamm 27:932-936

14. Putzu C, Cortinovis DL, Colonese F et al (2018) Blood cell count indexes as predictors of outcomes in advanced non-small-cell lung cancer patients treated with nivolumab. Cancer Immunol Immunother 67:1349-1353

15. Paliogiannis P, Fois AG, Sotgia $S$ et al (2018) The neutrophil-tolymphocyte ratio as a marker of chronic obstructive pulmonary disease and its exacerbations: a systematic review and meta-analysis. Eur J Clin Invest 48:e12984

16. Paliogiannis P, Fois AG, Sotgia S (2018) Neutrophil to lymphocyte ratio and clinical outcomes in COPD: recent evidence and future perspectives. Eur Respir Rev 27:147

17. Mochimaru T, Ueda S, Suzuki Y et al (2019) Neutrophil-to-lymphocyte ratio as a novel independent predictor of severe exacerbation in patients with asthma. Ann Allergy Asthma Immunol 122:337-339

18. Raghu G, Collard HR, Egan JJ et al (2011) An official ATS/ERS/ JRS/ ALAT statement: idiopathic pulmonary fibrosis: evidencebased guidelines for diagnosis and management. Am J Respir Crit Care Med 183:788-824

19. Brusasco V, Crapo R, Viegi G et al (2005) Coming together: the ATS/ERS consensus on clinical pulmonary function testing. Eur Respir J 26:1-2

20. Qi Q, Zhuang L, Shen Y et al (2016) A novel systemic inflammation response index (SIRI) for predicting the survival of patients with pancreatic cancer after chemotherapy. Cancer 122:2158-2167

21. Fernandez IE, Eickelberg O (2012) New cellular and molecular mechanisms of lung injury and fibrosis in idiopathic pulmonary fibrosis. Lancet 380:680-688

22. King TE Jr, Albera C, Bradford WZ (2009) Effect of interferon gamma-1b on survival in patients with idiopathic pulmonary fibrosis (INSPIRE): a multicentre, randomised, placebo-controlled trial. Lancet 374:222-228

23. Raghu G, Brown KK, Bradford WZ et al (2004) A placebo-controlled trial of interferon gamma-1b in patients with idiopathic pulmonary fibrosis. N Engl J Med 350:125-133

24. Raghu G, Brown KK, Collard HR et al (2017) Efficacy of simtuzumab versus placebo in patients with idiopathic pulmonary fibrosis: a randomised, double-blind, controlled, phase 2 trial. Lancet Respir Med 5:22-32

25. Gregory AD, Kliment CR, Metz HE et al (2015) Neutrophil elastase promotes myofibroblast differentiation in lung fibrosis. J Leukoc Biol 98:143-152

26. Takemasa A, Ishii Y, Fukuda T (2012) A neutrophil elastase inhibitor prevents bleomycin-induced pulmonary fibrosis in mice. Eur Respir J 40:1475-1482

27. Mills CD (2012) M1 and M2 macrophages: oracles of health and disease. Crit Rev Immunol 32:463-488 
28. Schupp JC, Binder H, Jäger B et al (2015) Macrophage activation in acute exacerbation of idiopathic pulmonary fibrosis. PLoS ONE 10:e 0116775

29. Kolahian S, Fernandez IE, Eickelberg O et al (2016) Immune mechanisms in pulmonary fibrosis. Am J Respir Cell Mol Biol 55:309-322

30. Ziegenhagen MW, Zabel P, Zissel G et al (1998) Serum level of interleukin 8 is elevated in idiopathic pulmonary fibrosis and indicates disease activity. Am J Respir Crit Care Med 157:762-768

31. Beeh KM, Beier J, Kornmann O et al (2003) Neutrophilic inflammation in induced sputum of patients with idiopathic pulmonary fibrosis. Sarcoidosis Vasc Diffuse Lung Dis 20:138-143

32. Tsoutsou PG, Gourgoulianis KI, Petinaki E et al (2004) ICAM1, ICAM-2 and ICAM-3 in the sera of patients with idiopathic pulmonary fibrosis. Inflammation 28:359-364

33. Daniil Z, Kitsanta P, Kapotsis G et al (2005) CD8+ T lymphocytes in lung tissue from patients with idiopathic pulmonary fibrosis. Respir Res 6:81

34. Nishimura S, Nagasaki M, Kunishima S et al (2015) IL-1alpha induces thrombopoiesis through megakaryocyte rupture in response to acute platelet needs. J Cell Biol 209:453-466
35. Yáñez A, Coetzee SG, Olsson A et al (2017) Granulocyte-monocyte progenitors and monocyte-dendritic cell progenitors independently produce functionally distinct monocytes. Immunity 47:890-902

36. Satoh T, Nakagawa K, Sugihara F et al (2017) Identification of an atypical monocyte and committed progenitor involved in fibrosis. Nature 541:96-101

37. Chen L, Kong X, Wang Z et al (2020) Pretreatment systemic inflammation response index in patients with breast cancer treated with neoadjuvant chemotherapy as a useful prognostic indicator. Cancer Manag Res 12:1543-1567

38. Pacheco-Barcia V, Mondéjar Solís R, France T et al (2020) A systemic inflammation response index (SIRI) correlates with survival and predicts oncological outcome for mFOLFIRINOX therapy in metastatic pancreatic cancer. Pancreatology 20:254-264

Publisher's Note Springer Nature remains neutral with regard to jurisdictional claims in published maps and institutional affiliations. 